\title{
Down-Regulation of Sox11 Is Required for Efficient Osteogenic Differentiation of Adipose-Derived Stem Cells
}

\author{
Mi Kyung Choi ${ }^{1}$, Ikjoo Seong ${ }^{1,2}$, Seon Ah Kang ${ }^{1}$, and Jaesang Kim ${ }^{1,2, *}$
}

\begin{abstract}
Adipose-derived stem cells represent a type of mesenchymal stem cells with the attendant capacity to self-renew and differentiate into multiple cell lineages. We have performed a microarray-based gene expression profiling of osteogenic differentiation and found that the transcription factor Sox11 is down-regulated during the process. Functional assays demonstrate that down-regulation of Sox11 is required for an efficient differentiation. Furthermore, results from forced expression of constitutively-active and dominant-negative derivatives of Sox11 indicate that Sox11 functions as a transcriptional activator in inhibiting osteogenesis. Sox11 thus represents a novel regulator of osteogenesis whose expression and activity can be potentially manipulated for controlled differentiation.
\end{abstract}

\section{INTRODUCTION}

Mesenchymal stem cells have been isolated from various tissues and organs including bone marrow, adipose tissue, peripheral blood, umbilical cord blood, skeletal muscle, lung and heart (Beltrami et al., 2003; Griffiths et al., 2005; Jiang et al., 2002; Kuznetsov et al., 2001; Wagner et al., 2005). Among these, adipose-derived stem cells (ADSCs) are notable for the high accessibility and abundance with minimum morbidity during the harvest procedure. ADSCs are capable of differentiating into multiple mesodermal cell types including chondrocytes, adipocytes, and osteoblasts (Konno et al., 2013). They have also been reported to be able to generate non-mesodermal lineage cell types including neurons and hepatocytes (Konno et al., 2013). Although multipotency of ADSCs is well documented, the detailed mechanisms through which key regulators control self-renewal and differentiation are far from clear. In particular,

${ }^{1}$ Department of Life Science, Ewha Womans University, Seoul 120-750, Korea, ${ }^{2} E$ wha Research Center for Systems Biology, Seoul 120-750, Korea

*Correspondence: jkim1964@ewha.ac.kr

Received 29 January, 2014; revised 6 March, 2014; accepted 7 March, 2014; published online 7 April, 2014

Keywords: adipose-derived stem cell, differentiation, mesenchymal stem cell, osteogenesis, Sox 11 unlike embryonic stem cells or neural stem cells, transcriptional regulators that endow the essential 'stemness' property of ADSCs which must be down-regulated for efficient differentiation to occur are not well-defined.

Sox genes constitute a family of transcription factors whose defining feature is the presence of high-mobility-group (HMG) box, a conserved 80-85 amino acid-long domain that binds to the minor groove of DNA in a sequence-specific manner (Lee et al., 2008; Wissmuller et al., 2006). Sox transcription factors play critical roles in diverse development and differentiation programs and in particular have been shown to be key constitutive elements of 'stemness' property in multiple types of stem cells (Sarkar and Hochedlinger, 2013). For example, Sox2 is a defining transcription factor of embryonic stem (ES) cells along with Oct3/4 (Takahashi and Yamanaka, 2006). Sox1, Sox2, and Sox3 are expressed in neural stem cells and inhibit neuronal differentiation (Bylund et al., 2003). Sox10 maintains multipotency and inhibits neuronal differentiation of neural crest stem cells (Kim et al., 2003).

Although expressed in a highly dynamic manner throughout embryogenesis, the only activity of Sox11 known in detail is promotion of neuronal differentiation (Bergsland et al., 2006). It is also believed to be involved in nerve regeneration and oligodendrocyte differentiation (Jing et al., 2012; Kuhlbrodt et al., 1998; Salerno et al., 2012). Here, we show that Sox11 is expressed in ADSCs and is down-regulated during osteogenic differentiation. Importantly, Sox11 functions as a transcriptional activator, and its down-regulation is necessary for efficient osteogenic differentiation. Sox11 thus represents a candidate regulatory target and a potential molecular tool for the control of ADSCs in vitro.

\section{MATERIALS AND METHODS}

\section{Isolation and culture of mouse ADSCs}

The ADSCs were prepared from 6 weeks old male C57BL/6 mice. Subcutaneous fat tissue was isolated from inguinal and dorsal parts of mice. After mincing with scissors, tissue fragments were incubated in PBS with $0.075 \%$ type 2 collagenase (SIGMA) and $2 \%$ BSA (AMRESCO) for $2 \mathrm{~h}$ at $37^{\circ} \mathrm{C}$. The ADSCs were subsequently isolated by centrifugation and incubated in $160 \mathrm{mM} \mathrm{NH}_{4} \mathrm{Cl}$ for $10 \mathrm{~min}$ at $\mathrm{RT}$ to remove contaminating erythrocytes. After filtration with $100 \mu \mathrm{m}$ nylon cell strainer (BD Falcon), ADSCs were centrifuged, re-suspended and 
Sox11 in Osteogenic Differentiation of ADSC

Mi-Kyung Choi et al.

Table 1. Primers for RTPCR

\begin{tabular}{lccc}
\hline Genes & Forward & Reverse & Size(bp) \\
\hline Dsip & caccgaaatgtatcagacc & gttcttctcaagcagctcac & 252 \\
Sox5 & ctggagctcagagtcaaga & cctgctcctcatagtatgg & 235 \\
Klf15 & caagaacccagcagcagaac & cttcacacccgagtgagatc & 208 \\
Klf13 & cagaggaagcacaagtgcc & agcgtgcctgggtggaagtt & 289 \\
Sox11 & ctggtggataaggacctgga & cgcctctcaatacgtgaaca & 153 \\
GAPDH & aaggtcatcccagagctgaa & tgctgtagccaaattcgttg & 300 \\
\hline
\end{tabular}

All sequences are shown in the $5^{\prime} \rightarrow 3^{\prime}$ direction.

plated in DMEM-F12 medium (Invitrogen) with 10\% FBS and $1 \%$ streptomycin/penicillin at $37^{\circ} \mathrm{C}$ with $5 \% \mathrm{CO}_{2}$. The culture medium was changed after $20 \mathrm{~min}$ to remove non-adherent cells. Cells were typically amplified for 3 days and re-plated at a density of $5 \times 10^{4}$ cells per well in 24 -well plates. All the mouse experiments were carried out in accordance with the animal protocol approved by the Animal Care Committee of Ewha Laboratory Animal Genomics Center.

\section{Flow cytometry analysis}

ADSCs were trypsinized, washed and suspended in $100 \mu \mathrm{l}$ of ice-cold PBS containing 0.5\% BSA. Rat anti-mouse antibodies (FITC-CD106, FITC-Ly-6A/E (Sca-1), PE-CD45, PE-CD44, and PE-CD11b; BD Pharmingen) were added for 30 min incubation at RT. PE-conjugated Rat IgG2b and FITC-conjugated Rat IgG2a (BD Pharmingen) were used as controls at concentrations recommended by the manufacturer. The cells were analyzed by flow cytometry using FACSCalibur (BD Biosciences) with the BD Cell-Quest_ Pro version 4.0.1 software (BD Biosciences).

\section{Osteogenic differentiation}

ADSCs were re-plated in 24-well plate at a density of $5 \times 10^{4}$ cells per well. After overnight culture, the osteogenic medium (DMEM-F12, $50 \mu \mathrm{M}$ L-ascorbic acid, $10 \mathrm{mM} \beta$-glycerophosphate, $0.1 \mu \mathrm{M}$ dexamethasone, $0.4 \%$ DMSO, 15\% FBS, $1 \%$ streptomycin/penicillin) was applied, and the culture was continued for 14 days. The osteogenic medium was replaced every 2-3 days.

\section{Microarray expression profiling}

Total RNA was extracted using Trizol (Invitrogen) and purified using RNeasy columns (Qiagen) according to the manufacturers' protocol. To generate biotinylated cRNA, total RNA was amplified and purified using the Ambion Illumina RNA amplification kit (Ambion) according to the manufacturer's instructions. The $1.5 \mu \mathrm{g}$ of labeled cRNA samples were hybridized to mouse- 6 expression bead array for $16-18 \mathrm{~h}$ at $58^{\circ} \mathrm{C}$ (Illumina, Inc.), and detection of array signal was carried out using Amersham fluorolink streptavidin-Cy3 (GE Healthcare Bio-Sciences) following the bead array manual. Arrays were scanned with an Illumina bead array Reader confocal scanner, and array data export processing and analysis were performed using Illumina BeadStudio v3.1.3 (Gene Expression Module v3.3.8). For the fold-change comparative analysis, it was arbitrarily determined that genes of significance in osteogenesis should show over 2fold change in expression at least at one time point compared to the control sample ( $t=0$ : before osteogenic induction) which led to 4223 significant probes. The list of the probes/genes is provided as supplementary data. The K-means clustering method $(k=9)$ was used to classify these probes according to characteristic temporal expression patterns. Ontology-based analyses were performed using Panther database (http://www.pantherdb.org). Details of the experimental methods and analyses are available upon request. The complete microarray data were deposited in the Gene Expression Omnibus (GEO) database [GEO: GSE34842]. All data are MIAME compliant.

\section{RTPCR}

Total RNA was extracted from mouse ADSCs using Qiagen RNA extraction kit (Qiagen). RNA was reverse-transcribed using SuperScript First-Strand Synthesis System for RT-PCR kit (Invitrogen), and PCR was performed to confirm results from the microarray assay. The genes and primer sequences are provided (Table 1). For real time RT-PCR, the mRNA levels were measured in duplicates with SYBR GREEN master mix (Applied Biosystems) using ABI PRISM 7300 (Applied Bioystems) and normalized by GAPDH expression level.

\section{Preparation of retroviruses}

To isolate a DNA fragment encompassing the complete open reading frame (ORF) of mouse Sox11, PCR amplification was carried out using a BAC clone (RP23-285B14, Children's Hospital Oakland Research Institute) as the template, and the resulting product was ligated to pGEM-T Easy vector plasmid using TA Cloning kit (Promega). Sox11 HMG box was PCRamplified from the full ORF plasmid. VP16 activator domain was PCR-amplified from a plasmid containing a GAL4-VP16 fusion gene (kind gift of WJ Lee) and ligated to the HMG box. Engrailed Repressor (EnR) domain was PCR-amplified from a plasmid containing full length Engrailed gene (kind gift of WJ Lee) with a $3^{\prime}$ oligonucleotide primer encoding myc-epitope tag in addition to the C-terminus of Engrailed and ligated to the HMG box. Each of the DNA fragments containing full Sox11 ORF, HMG box-VP16, and HMG box-EnR was cloned into pLZRS vector $5^{\prime}$ to pre-inserted IRES-GFP (Kim et al., 2003). The control virus (GFP virus) had just the IRES nuclear GFP in $\mathrm{pLZRS}$. The sequences of oligonucleotide primers used for cloning and details of the cloning procedure are available upon request.

The shRNA targeting Sox11 and control shRNA containing 5 alternate nucleotides (for the nucleotide sequences, see Fig. 4) were designed to be contained within miRNA scaffold (miRshRNA) (Stegmeier et al., 2005) and synthesized as long duplex oligonucleotides (Bioneer Inc.) which were subsequently ligated to pLZRS vector $5^{\prime}$ to IRES-GFP. Preparation of hightiter virus was carried out as described with minor modifications (Kim et al., 2003; Ory et al., 1996). Further details of construction of viral vectors and generation of pseudotyped viral particles will be provided upon request. 
A

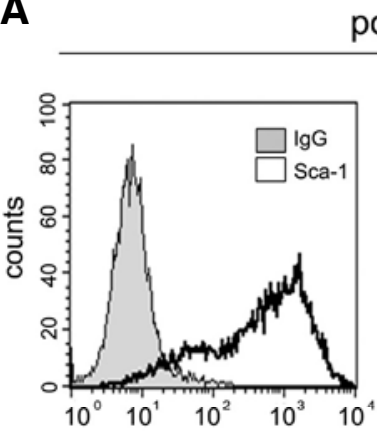

positive
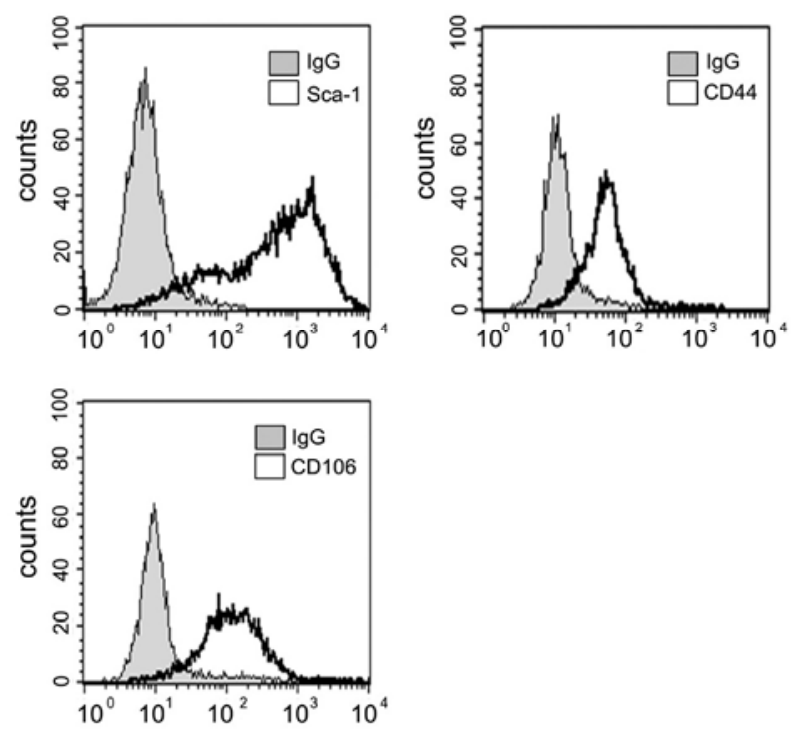

negative
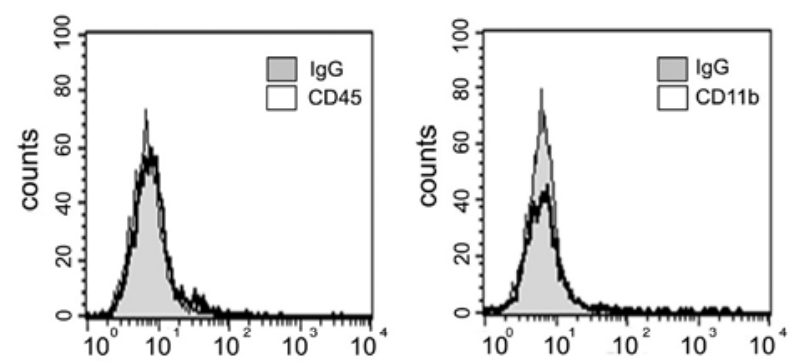

B

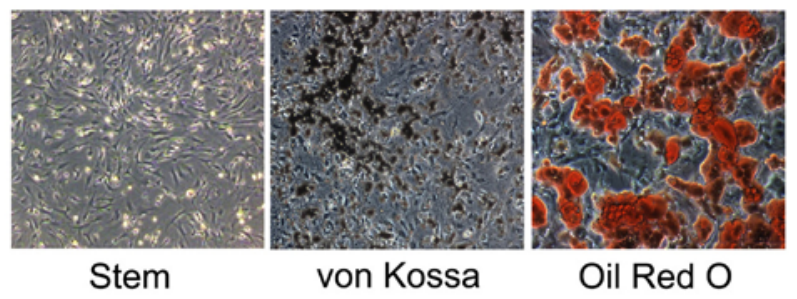

Fig. 1. Characterization of ADSCs. (A) Flow cytometric analysis of ADSCs. Cells were stained with monoclonal antibodies against indicated surface markers or isotype-matched control antibodies and subjected to flow cytometric analyses. (B) Multipotency of ADSCs. Representative microscopic visual fields are shown for freshly prepared ADSCs (left), von Kossa stained cells after 14 days of culture under osteogenic differentiation condition (middle), and Oil Red $O$ stained cells after 6 days of adipogenic condition (right).

von Kossa staining

Cells were washed twice with PBS and fixed with $70 \%$ ethanol for $30 \mathrm{~min}$ at RT. After a brief wash with distilled water, cells were stained with $5 \%$ silver nitrate solution (Spectrum Chemicals \& Laboratory Products) until calcium deposits turned black. Cells were washed twice with distilled water, and mineralization of the cells was observed using bright field microscopy.

Alizarin Red staining and quantitation of mineralization Cells typically in 24-well plates were rinsed twice with PBS and fixed with $70 \%$ ethanol for 30 min at RT. After a brief wash with distilled water, cells were stained with $40 \mathrm{mM}$ alizarin red at $\mathrm{pH}$ 4.1 (Junsei Chemical) for $30 \mathrm{~min}$ at RT with gentle shaking. After washing with distilled water, cells were incubated with 400 $\mu \mathrm{l}$ of $10 \%$ acetic acid for $30 \mathrm{~min}$ at RT with gentle shaking. The extracted alizarin red solution was transferred to a new tube and neutralized with $100 \mu \mathrm{l}$ of $10 \%$ ammonium hydroxide. $\mathrm{OD}_{405}$ was determined on duplicate samples to quantitate mineralization.

\section{Adipogenic differentiation and Oil Red O staining}

Cells were plated at a density of $5 \times 10^{4}$ per well in 24-well plates. After overnight incubation, adipogenic induction medium with $5 \mu \mathrm{g} / \mathrm{ml}$ insulin (SIGMA), $1 \mu \mathrm{M}$ Dexamethason (SIGMA), $500 \mu \mathrm{M}$ 3-isobutyl-1-methylxanthine (IBMX), $1 \mu \mathrm{g} / \mathrm{ml}$ rosiglitazone (ENZO Lifesciences) in DMEM-F12 was applied for 2 days. Subsequently, cells were cultured for another 2 days in DMEM-F12 with $5 \mu \mathrm{g} / \mathrm{ml}$ insulin, 10\% FBS, 1\% streptomycin/penicillin and for 2 more days in the medium without insulin. For Oil Red O staining, cells were rinsed twice with PBS and fixed with $10 \%$ formalin in PBS for 5 min at RT. Cells were subsequently fixed for $1 \mathrm{~h}$ at RT with fresh formalin solution, washed with $60 \%$ isopropanol and then completely dried. Oil
Red O working solution, made up of 6 parts filtered Oil Red O stock (0.7 g Oil Red O in $200 \mathrm{ml}$ isopropanol; Sigma) and 4 parts distilled water, was applied to the dried well for $10 \mathrm{~min}$ incubation. Cells were rinsed with distilled water 4 times and dried prior to microscopic observation.

\section{RESULTS}

In preparing mouse ADSCs, we followed published protocols with minor modifications (De Ugarte et al., 2003; Yamamoto et al., 2007). ADSCs were examined based on two criteria, expression of specific cell-surface markers and capacity to differentiate into osteoblasts and adipocytes. Prepared ADSCs were subjected to flow cytometric analyses and shown to be positive for Ly-6A/E (Sca-1), CD44, and CD106 and negative for CD45 and CD11b (Fig. 1A). Such expression pattern is fully consistent with results from several previous studies (Schaffler and Buchler, 2007; Sun et al., 2003). Cells were also cultured under osteogenic and adipogenic conditions and examined by von Kossa and Oil Red O staining procedures respectively (Fig. $1 \mathrm{~B})$. The results confirmed that cells were capable of differentiating into osteoblasts and adipocytes consistent with the population-level multipotency of ADSCs (Konno et al., 2013).

We carried out a microarray-based expression profiling of osteogenic differentiation of ADSCs using a standard culture condition. Cells were harvested at various time points between 0 and 14 days, and the labeled cRNA probe converted from the extracted RNA was subsequently applied to high-density microarray chips (see "Materials and Methods"). We noted that several well-established osteoblast markers including osteopontin (Id Boufker et al., 2011; McGee-Lawrence et al., 2011), osteoglycin (Guntur et al., 2012; Huttunen et al., 2008), periostin (Alonso et al., 2008; Zhu et al., 2009), osteonectin (Curran 
A

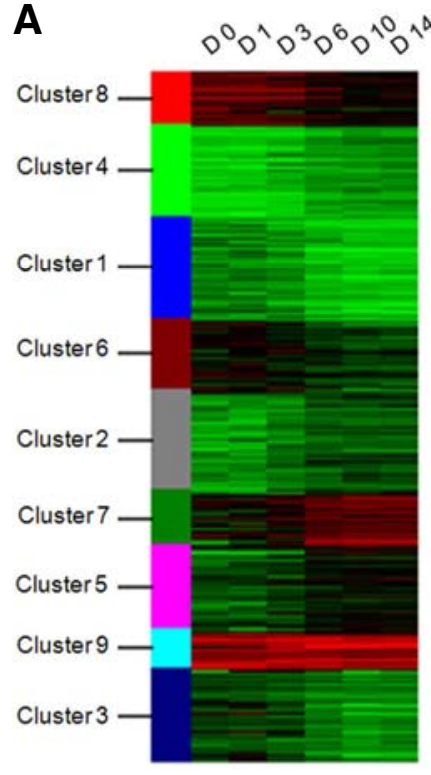

B

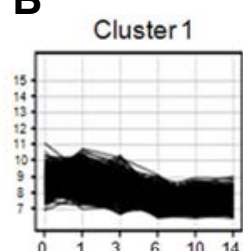

Cluster 4

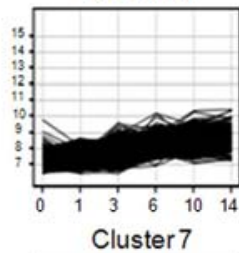

Cluster 7

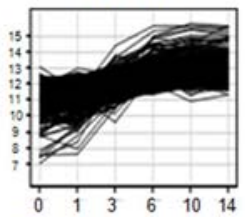

Cluster 2

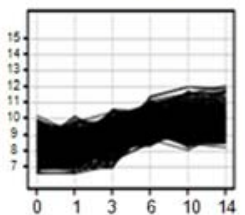

Cluster 5

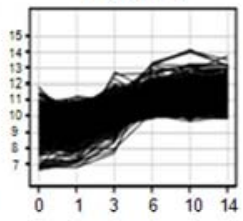

Cluster 8

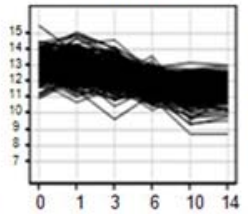

C

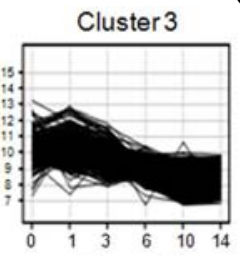

Cluster 6

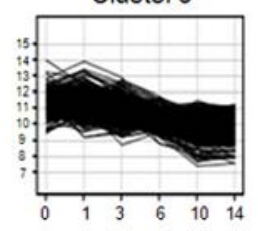

Cluster 9

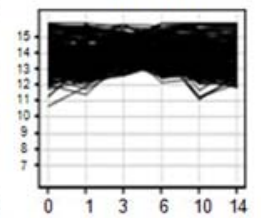

$\begin{array}{llll}0 & 3 & 10 & 14\end{array}$

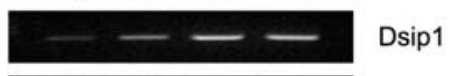

$\longrightarrow-\ldots$ Sox5
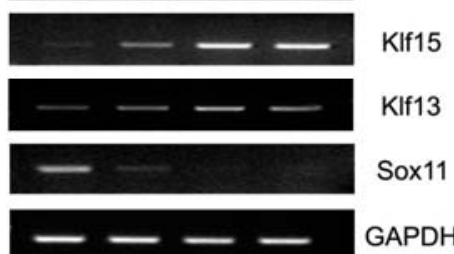

Fig. 2. Expression profiling with microarray. (A) Heat-map showing dynamic expression of representative genes from each cluster during osteogenesis. Red and green colors respectively represent high and low levels of expression. (B) Clustering analysis of the microarray data. 4223 probes that showed over 2 fold change in expression at least at one of the time points are clustered into 9 groups using K-means clustering method (list provided in the Supplementary Table 1). (C) RTPCR was carried out with cDNA preparations from the indicated days of osteogenic induction to confirm results from microarray assay. GAPDH is used as the control.

A

\section{Control}

Sox11
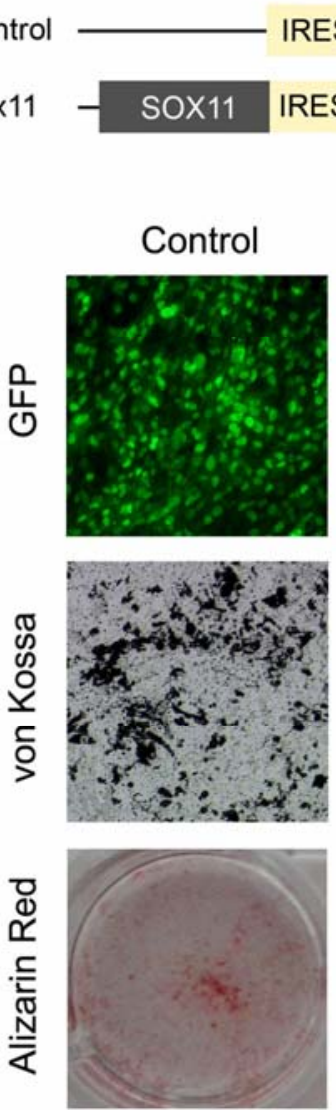

Sox11
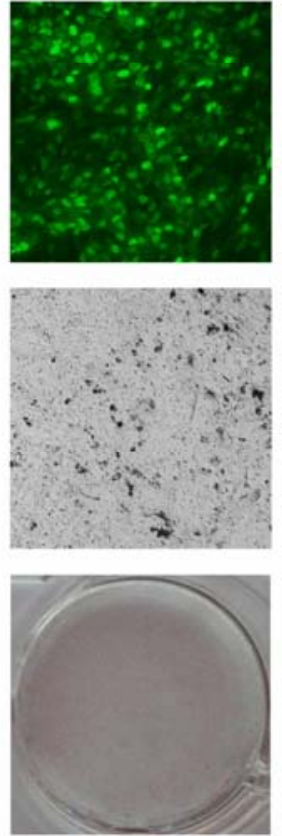

C

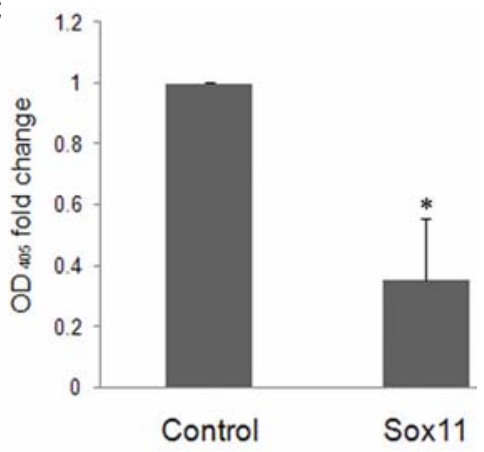

Fig. 3. Sox11 gain-of-function assay. (A) Expression vectors are schematically illustrated. NLS stands for nuclear localization signal. (B) ADSCs infected with Sox11 or control pLZRS viruses are examined for infection by GFP expression (upper panels) and for osteogenesis by von Kossa staining (middle panels) and Alizarin Red staining (lower panels). Typical results from multiple trials are shown. (C) Alizarin red staining is extracted and quantitated by measuring $\mathrm{OD}_{405}$. Normalized relative levels of $\mathrm{OD}_{405}$ are shown with the value from the control virus infection as 1 (i.e. 100\%). Data are the average of seven independent experiments, and the error bar represents the standard deviation. A significant difference $\left({ }^{*} p<0.001\right)$ was noticed. 
A

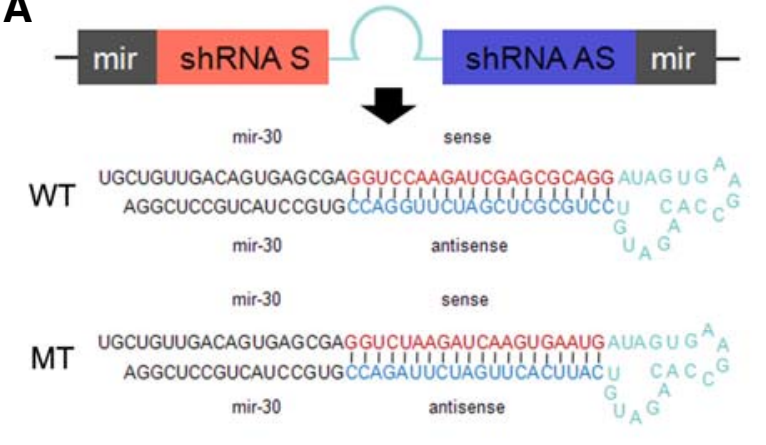

C
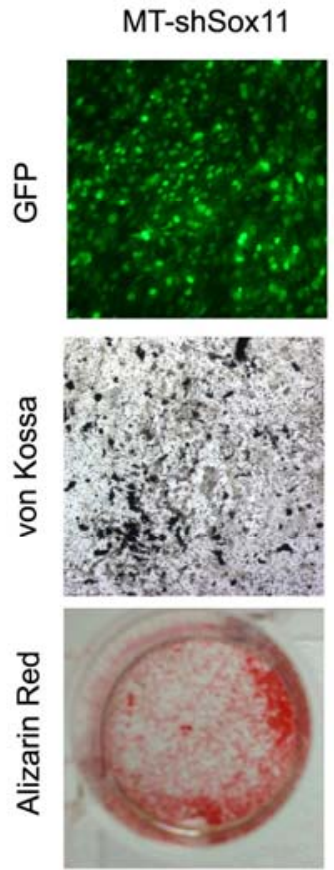

WT-shSox11
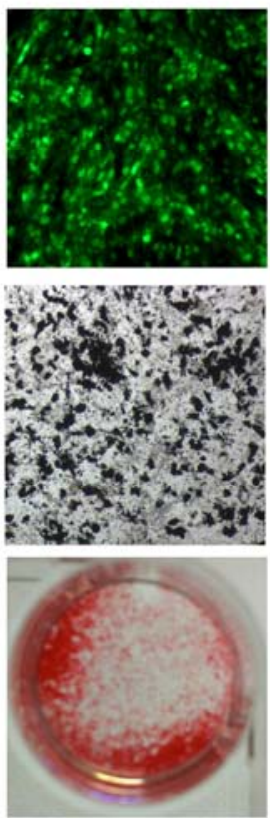

B
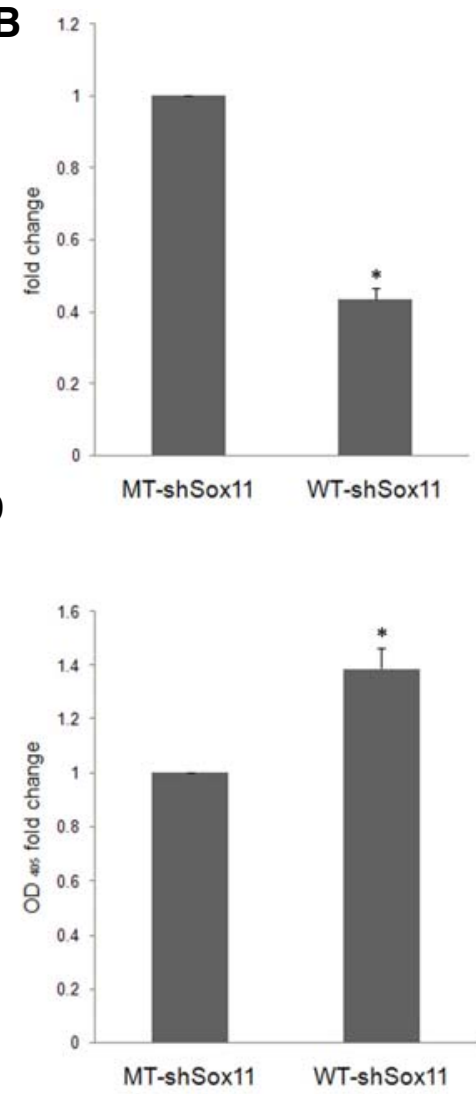

Fig. 4. Sox11 loss-of-function assay. (A) Schematic diagram of miR-shRNA is shown at the top. $S$ and $A S$ stand for sense and antisense, respectively. Sequences and presumptive hairpin structures are shown for mature miR-shRNA specifically targeting Sox11 (WT-shSox11; WT for wild type) and control miR-shRNA (MT-shSox11; MT for mutant). WT-shSox11 differs from MT-shSox11 at 5 nucleotide positions. (B) Real time RTPCR was carried out to test the effect of WT-shSox11. Relative levels of Sox11 normalized to GAPDH are shown with the value from the MT-shSox11 control virus as 1 (i.e. 100\%). Data are the average of three independent experiments, and the error bar represents the standard deviation. A significant difference $\left({ }^{*} p<0.05\right)$ was noticed. (C) ADSCs infected with Sox11-targeting shRNA or control shRNA pLZRS viruses are examined for infection by GFP expression (upper panels) and for osteogenesis by von Kossa staining (middle panels) and Alizarin Red staining (lower panels). Typical results from multiple trials are shown. (D) Alizarin Red staining is extracted and quantitated by measuring $\mathrm{OD}_{405}$. Normalized relative levels of $\mathrm{OD}_{405}$ are shown with the value from the control virus infection as 1 (i.e. 100\%). Data are the average of three independent experiments, and the error bar represents the standard deviation. A significant difference $\left({ }^{*} \mathrm{p}<0.05\right)$ was noticed.

et al., 2006; Pre et al., 2011), ocil (Quan et al., 2009), and osteomodulin (Patel et al., 2007) whose expression is known to be induced during osteogenesis showed significant upregulation in the microarray screen supporting the validity of the expression profile. The complete microarray data have been deposited in the Gene Expression Omnibus (GEO) database [GEO: GSE34842]. Genes showing dynamic expression pattern were annotated and clustered for systematic functional analyses (Figs. 2A and 2B). The list of 4223 probes, their cluster designation and fold changes are provided (Supplementary Table S1). We performed a semi-quantitative RTPCR assay to confirm the results from the microarray assay on several transcription factors and found that osteogenic differentiation indeed led to changes in the expression of these genes in a consistent pattern (Fig. 2C). Specifically, the expression levels of Dsip1, Sox5, Klf5, and KIf3 increased while that of Sox11 decreased.

Overrepresentation analysis for Gene Ontology (GO) terms of biological process was carried out on each of the gene clusters using DAVID Bioinformatics Resources 6.7 (Huang da et al., 2009). Only the clusters 2 and 3 contained significantly enriched GO terms $(P$ value $<0.005$ after multiple testing correction using the Benjamini-Hochberg method; Supplementary Table S2). Interestingly, the genes up-regulated belonging to the cluster 2 were those known to be responsive to endogenous and exogenous stimuli including various organic substances and hormones suggesting that osteogenesis is initiated in large part by cell-extrinsic stimuli. In contrast, down-regulated genes found in the cluster 3 mostly have to do with cell cycle progression indicating that osteogenic differentiation of ADSCs requires concurrent cessation of cell division.

Subsequent studies were focused on Sox11. We noted that Sox11 belonged to cluster 1 which encompasses downregulated genes and hypothesized that like other members of the same gene family, its down-regulation is important for terminal differentiation. We chose pLZRS retroviral vector for gainand loss-of-function experiments (Kinsella and Nolan, 1996; Ory et al., 1996). We were able to achieve co-expression of Sox11 and GFP by constructing a bicistronic vector with the use of internal ribosome entry site (IRES) 5 ' to GFP, and for the 
A

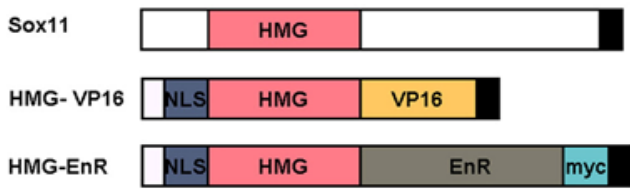

C

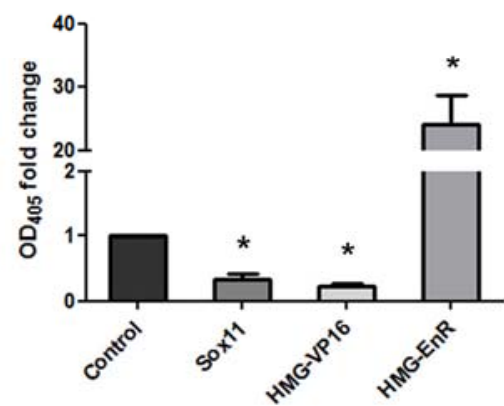

B
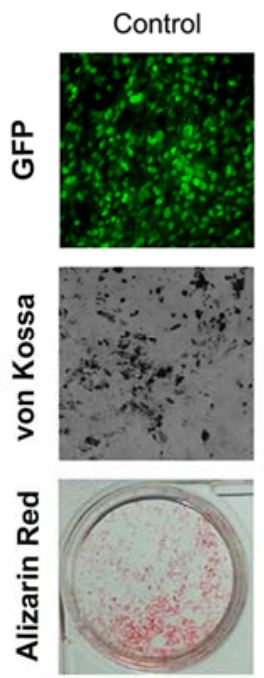

Sox11
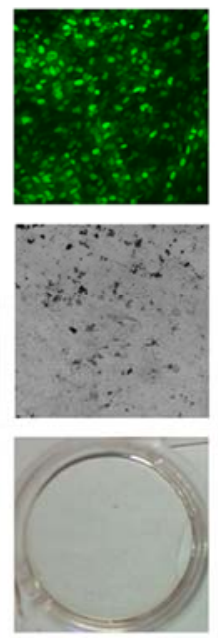

HMG-VP16
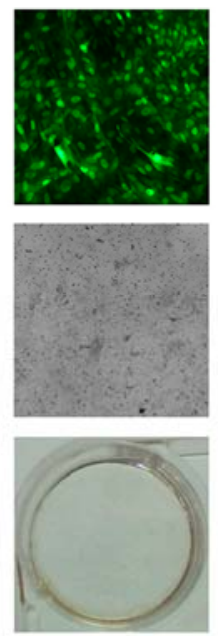

HMG-EnR
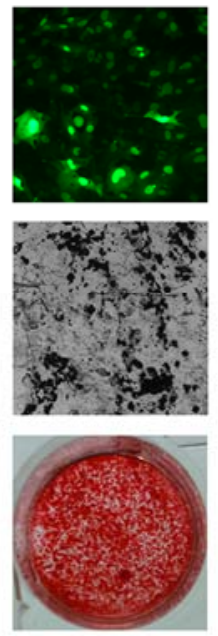

Fig. 5. Mechanism of Sox11 activity. (A) Sox11 derivatives are schematically illustrated. NLS in the N terminus stands for nuclear localization signal, and myc in the $C$ terminus indicates the presence of a myc-epitope. (B) ADSCs infected with indicated viruses are examined for infection by GFP expression (upper panels) and for osteogenesis by von Kossa staining (middle panels) and Alizarin Red staining (lower panels). Typical results from multiple trials are shown. (C) Alizarin Red staining is extracted and quantitated by measuring $\mathrm{OD}_{405}$. Normalized relative levels of $\mathrm{OD}_{405}$ are shown with the value from the control virus infection as 1 (i.e. 100\%). Data are the average of 6 independent experiments, and the error bars represent the standard deviation. A significant difference $\left({ }^{*} p<0.05\right)$ was noticed.

control virus, we used pLZRS vector with IRES-GFP only (Fig. $3 A)$. After induction and concentration of retroviruses, we were routinely able to infect over $90 \%$ of ADSCs as judged by GFP expression (Fig. 3B). ADSCs were infected with Sox11 and control viruses and examined for osteogenic differentiation by von Kossa staining and Alizarin Red staining after 14 days of culture. It was readily noticed that ectopic expression of Sox11 significantly inhibited osteogenic differentiation (Fig. 3B). The extent of mineralization was quantitated by spectrophotometric measurement of the extracted Alizarin Red stain, and it was seen that in agreement with the visual observation after von Kossa staining, Sox11 expression led to a significant inhibition of osteogenic differentiation (Fig. 3C).

We also sought to examine the effect of down-regulation of Sox11 on osteogenesis. To this end, a pLZRS vector designed to express an shRNA embedded in a microRNA context (miRshRNA) that specifically targets Sox 11 was constructed (Stegmeier et al., 2005). For the control, we generated a vector that expresses a mutant shRNA whose nucleotide sequence differs from that of the wild type at 5 positions (Fig. 4A). ADSCs were prepared and infected with shRNA viruses, and it was seen that the wild type shRNA led to reduction in Sox11 expression to approximately $40 \%$ of the level seen with the control shRNA (Fig. 4B). Osteogenesis as judged by von Kossa staining and Alizarin Red staining showed a somewhat subtle but consistent enhancement by Sox11 knockdown (Fig. 4C). Quantitative analysis with Alizarin Red extract confirmed the result (Fig. 4D and see "Discussion").

We next attempted to address whether Sox11 functions as a transcriptional activator or repressor in inhibiting osteogenic differentiation. Two derivatives of Sox11 were constructed for this purpose: a constitutively active derivative containing VP16 activator domain in tandem with the HMG-box of Sox11 and a constitutive repressor derivative containing Engrailed Repres- sor domain in tandem with the HMG-box of Sox11 (Fig. 5A). Viruses expressing these proteins were used to infect ADSCs along with the control and full-length Sox11 viruses described above. Upon induction, we noticed that the full-length Sox11 and HMG-VP16 led to inhibition of osteogenesis compared to the control while the reverse was observed with HMG-EnR derivative (Fig. 5B). That Sox11 behaves like HMG-VP16, a constitutive activator, indicates that Sox11 functions in ADSCs as a transcriptional activator. It is also noted that the constitutive repressor derivative, HMG-EnR had a particularly strong stimulatory effect on osteogenesis (over 20 fold by Alizarin red staining; Fig. 5C). Taken together, these results indicate that Sox11 is actively promoting expression of a group of genes, a subset of which antagonizes osteogenesis of ADSCs (see "Discussion").

\section{DISCUSSION}

Cellular differentiation often results from induction and/or inhibition of transcriptional regulatory activities. This generalization most likely applies to differentiation of diverse types of stem cells into mutilple cell lineages including osteogenic differentiation of ADSCs. Results from the microarray assay provided in this study covering various stages of osteogenesis should thus represent a robust data set for dissection of this process. Our focus in this study was Sox11, a member of the Sox transcription factor gene family which features prominently as regulators of self-renewal and differentiation of multiple types of stem cells (Sarkar and Hochedlinger, 2013). Importantly, we showed in this study that Sox11 is a transcription factor whose downregulation is required for efficient osteogenic differentiation. To the best of our knowledge this is the first report on such function of Sox11. While forced maintenance of Sox11 had a clear effect, knock-down led to a relatively subtle effect. This is likely due to that Sox11 is rapidly down-regulated by induction of 
osteogenic differentiation which would limit the effect of Sox11 knock-down.

Recently, Gadi and coworkers reported that Sox11 enhances early osteoblast differentiation of bone marrow mesenchymal cells (BMScs) (Gadi et al., 2013). In fact, in these cells, Sox11 expression was absent initially, induced by osteogenic differentiation and subsequently subsided prior to full osteogenic differentiation. This is clearly in contrast to the case of ADSCs which showed a high-level Sox11 expression at the stem cell stage which was only down-regulated therefrom during osteogenic differentiation. Even in BMScs, down-regulation of Sox11 may be required for terminal differentiation, but this possibility was not examined in full detail (Gadi et al., 2013). Such difference between ADSCs and BMScs suggests that the two cell types are distinct at the molecular level and that refined strategies in inducing differentiation into various progeny cell types may be required.

Results obtained from ectopic expression of HMG-EnR are worthy of further elaboration. First, the constitutive repressor derivative, HMG-EnR, led to a dramatic induction of osteogenesis which is opposite to the effect of forced expression of Sox11 or HMG-VP16, a constitutive activator derivative. The result therefore indicates that Sox11 is a transcriptional activator and performs its role in ADSCs by directly activating the expression of ADSC-specific target genes rather than repressing genes involved in osteogenic differentiation. Secondly, presumptive target genes must be co-activated by other transcription factors than Sox11 in ADSCs as HMG-EnR expression showed a far greater effect than Sox11 knock-down. This may be one of the reasons for the relatively modest effect we saw with shRNA-mediated inhibition of Sox11 which would simply result in reduced transcriptional activation but not in repression. Finally, target genes of activation by Sox 11 must in turn be acting as direct or indirect repressors of osteogenesis, and isolating direct targets of Sox11 and dissecting the downstream network thus will likely yield further insights into the osteogenesis of ADSCs. A caveat is that HMG-EnR may not be as specific as the wild type Sox11 as domains outside the HMG box is missing. However, much of the specificity of Sox genes is known to reside within the HMG box which actively associates with partner proteins (Wilson and Koopman, 2002). At any rate, the specificity of HMG box from various Sox genes in terms of target recognition should be an important issue to be addressed down the road.

Transcription factors are currently the primary genetic tools for controlled differentiation. Their utility is dramatically demonstrated in the case of induced pluripotent stem cells (Takahashi and Yamanaka, 2006). In the future, activating or repressing derivatives of transcription factors as well as siRNAs and miRNAs that target transcription factors are likely to be part of the arsenal. HMG-EnR used in this study was particularly effective in promoting osteogenesis raising the possibility of using similar derivatives as tools for the programmed cellular differentiation.

Note: Supplementary information is available on the Molecules and Cells website (www.molcells.org).

\section{ACKNOWLEDGMENTS}

This study was supported by a grant of the Korea Health technology R\&D Project, Ministry of Health \& Welfare, Republic of Korea (A120262).

\section{REFERENCES}

Alonso, M., Claros, S., Becerra, J., and Andrades, J.A. (2008). The effect of type I collagen on osteochondrogenic differen-tiation in adipose-derived stromal cells in vivo. Cytotherapy 10, 597-610.

Beltrami, A.P., Barlucchi, L., Torella, D., Baker, M., Limana, F., Chimenti, S., Kasahara, H., Rota, M., Musso, E., Urbanek, K., et al. (2003). Adult cardiac stem cells are multipotent and support myocardial regeneration. Cell 114, 763-776.

Bergsland, M., Werme, M., Malewicz, M., Perlmann, T., and Muhr, J. (2006). The establishment of neuronal properties is controlled by Sox4 and Sox11. Genes Dev. 20, 3475-3486.

Bylund, M., Andersson, E., Novitch, B.G., and Muhr, J. (2003). Vertebrate neurogenesis is counteracted by Sox1-3 activity. Nat. Neurosci. 6, 1162-1168.

Curran, J.M., Chen, R., and Hunt, J.A. (2006). The guidance of human mesenchymal stem cell differentiation in vitro by controlled modifications to the cell substrate. Biomaterials 27 , 4783-4793.

De Ugarte, D.A., Morizono, K., Elbarbary, A., Alfonso, Z., Zuk, P.A. Zhu, M., Dragoo, J.L., Ashjian, P., Thomas, B., Benhaim, P., et al. (2003). Comparison of multi-lineage cells from human adipose tissue and bone marrow. Cells Tissues Organs 174, 101-109.

Gadi, J., Jung, S.H., Lee, M.J., Jami, A., Ruthala, K., Kim, K.M., Cho, N.H., Jung, H.S., Kim, C.H., and Lim, S.K. (2013). The transcription factor protein Sox11 enhances early osteoblast differentiation by facilitating proliferation and the survival of mesenchymal and osteoblast progenitors. J. Biol. Chem. 288, 25400-25413.

Griffiths, M.J., Bonnet, D., and Janes, S.M. (2005). Stem cells of the alveolar epithelium. Lancet 366, 249-260.

Guntur, A.R., Rosen, C.J., and Naski, M.C. (2012). N-cadherin adherens junctions mediate osteogenesis through $\mathrm{PIOK}$ signaling. Bone 50, 54-62.

Huang da, W., Sherman, B.T., and Lempicki, R.A. (2009). Systematic and integrative analysis of large gene lists using DAVID bioinformatics resources. Nat. Protoc. 4, 44-57.

Huttunen, M.M., Pekkinen, M., Ahlstrom, M.E., and Lamberg-Allardt, C.J. (2008). Long-term effects of tripeptide lle-Pro-Pro on osteoblast differentiation in vitro. J. Nutr. Biochem. 19, 708-715.

Id Boufker, H., Lagneaux, L., Fayyad-Kazan, H., Badran, B., Najar, M., Wiedig, M., Ghanem, G., Laurent, G., Body, J.J., and Journe, $F$. (2011). Role of farnesoid X receptor (FXR) in the process of differentiation of bone marrow stromal cells into osteoblasts. Bone 49, 1219-1231.

Jiang, Y., Vaessen, B., Lenvik, T., Blackstad, M., Reyes, M., and Verfaillie, C.M. (2002). Multipotent progenitor cells can be isolated from postnatal murine bone marrow, muscle, and brain Exp. Hematol. 30, 896-904.

Jing, X., Wang, T., Huang, S., Glorioso, J.C., and Albers, K.M. (2012). The transcription factor Sox11 promotes nerve regeneration through activation of the regeneration-associated gene Sprr1a. Exp. Neurol. 233, 221-232.

Kim, J., Lo, L., Dormand, E., and Anderson, D.J. (2003). SOX10 maintains multipotency and inhibits neuronal differentiation of neural crest stem cells. Neuron 38, 17-31.

Kinsella, T.M., and Nolan, G.P. (1996). Episomal vectors rapidly and stably produce high-titer recombinant retrovirus. Hum. Gene Ther. 7, 1405-1413.

Konno, M., Hamabe, A., Hasegawa, S., Ogawa, H., Fukusumi, T., Nishikawa, S., Ohta, K., Kano, Y., Ozaki, M., Noguchi, Y., et al. (2013). Adipose-derived mesenchymal stem cells and regenerative medicine. Dev. Growth Differ. 55, 309-318.

Kuhlbrodt, K., Herbarth, B., Sock, E., Enderich, J., HermansBorgmeyer, I., and Wegner, M. (1998). Cooperative function of POU proteins and SOX proteins in glial cells. J. Biol. Chem. 273, 16050-16057.

Kuznetsov, S.A., Mankani, M.H., Gronthos, S., Satomura, K., Bianco, P., and Robey, P.G. (2001). Circulating skeletal stem cells. J. Cell Biol. 153, 1133-1140.

Lee, K.E., Nam, S., Cho, E.A., Seong, I., Limb, J.K., Lee, S., and Kim, J. (2008). Identification of direct regulatory targets of the transcription factor Sox10 based on function and conservation. BMC Genomics 9, 408 .

McGee-Lawrence, M.E., McCleary-Wheeler, A.L., Secreto, F.J., 
Razidlo, D.F., Zhang, M., Stensgard, B.A., Li, X., Stein, G.S., Lian, J.B., and Westendorf, J.J. (2011). Suberoylanilide hydroxamic acid (SAHA; vorinostat) causes bone loss by inhibiting immature osteoblasts. Bone 48, 1117-1126.

Ory, D.S., Neugeboren, B.A., and Mulligan, R.C. (1996). A stable human-derived packaging cell line for production of high titer retrovirus/vesicular stomatitis virus G pseudotypes. Proc. Natl. Acad. Sci. USA 93, 11400-11406.

Patel, M.J., Liu, W., Sykes, M.C., Ward, N.E., Risin, S.A., Risin, D., and Jo, H. (2007). Identification of mechanosensitive genes in osteoblasts by comparative microarray studies using the rotating wall vessel and the random positioning machine. J. Cell Biochem. 101, 587-599.

Pre, D., Ceccarelli, G., Gastaldi, G., Asti, A., Saino, E., Visai, L., Benazzo, F., Cusella De Angelis, M.G., and Magenes, G. (2011) The differentiation of human adipose-derived stem cells (hASCs) into osteoblasts is promoted by low amplitude, high frequency vibration treatment. Bone 49, 295-303.

Quan, J.X., Zheng, F., Li, X.X., Hu, L.L., Sun, Z.Y., Jiao, Y.L., and Wang, B.L. (2009). Cloning and analysis of rat os-teoclast inhibitory lectin gene promoter. J. Cell Biochem. 106, 599-607.

Salerno, K.M., Jing, X., Diges, C.M., Cornuet, P.K., Glorioso, J.C., and Albers, K.M. (2012). Sox11 modulates brain-derived neurotrophic factor expression in an exon promoter-specific manner. J. Neurosci. Res. 90, 1011-1019.

Sarkar, A., and Hochedlinger, K. (2013). The sox family of trascription factors: versatile regulators of stem and progenitor cell fate. Cell Stem Cell 12, 15-30.

Schaffler, A., and Buchler, C. (2007). Concise review: adipose tissue-derived stromal cells--basic and clinical implications for novel cell-based therapies. Stem Cells 25, 818-827.
Stegmeier, F., Hu, G., Rickles, R.J., Hannon, G.J., and Elledge, S.J. (2005). A lentiviral microRNA-based system for single-copy polymerase II-regulated RNA interference in mam-malian cells. Proc. Natl. Acad. Sci. USA 102, 13212-13217.

Sun, S.K., Guo, Z.K., Xiao, X.R., Liu, B., Liu, X.D., Tang, P.H., and Mao, N. (2003). Isolation of mouse marrow mesen-chyma progenitors by a novel and reliable method. Stem Cells 21,527 535

Takahashi, K., and Yamanaka, S. (2006). Induction of pluripo-ent stem cells from mouse embryonic and adult fibroblast cultures by defined factors. Cell 126, 663-676.

Wagner, W., Wein, F., Seckinger, A., Frankhauser, M., Wirkner, U., Krause, U., Blake, J., Schwager, C., Eckstein, V., An-sorge, W., et al. (2005). Comparative characteristics of mesen- chymal stem cells from human bone marrow, adipose tissue, and umbilical cord blood. Exp. Hematol. 33, 1402-1416.

Wilson, M., and Koopman, P. (2002). Matching SOX: partner proteins and co-factors of the SOX family of transcriptional regulators. Curr. Opin. Genet. Dev. 12, 441-446.

Wissmuller, S., Kosian, T., Wolf, M., Finzsch, M., and Wegner, M. (2006). The high-mobility-group domain of Sox proteins interacts with DNA-binding domains of many transcription factors. Nucleic Acids Res. 34, 1735-1744.

Yamamoto, N., Akamatsu, $\mathrm{H}$., Hasegawa, S., Yamada, T., Na-ata, S., Ohkuma, M., Miyachi, E., Marunouchi, T., and Matsunaga, K. (2007). Isolation of multipotent stem cells from mouse adipose tissue. J. Dermatol. Sci. 48, 43-52.

Zhu, S., Barbe, M.F., Liu, C., Hadjiargyrou, M., Popoff, S.N., Rani, S., Safadi, F.F., and Litvin, J. (2009). Periostin-like-factor in osteogenesis. J. Cell Physiol. 218, 584-592. 